This item was submitted to Loughborough's Research Repository by the author.

Items in Figshare are protected by copyright, with all rights reserved, unless otherwise indicated.

Approximations to wave propagation through doubly-periodic arrays of scatterers

PLEASE CITE THE PUBLISHED VERSION

LICENCE

CC BY-NC-ND 4.0

REPOSITORY RECORD

Mclver, P.. 2019. "Approximations to Wave Propagation Through Doubly-periodic Arrays of Scatterers". figshare. https://hdl.handle.net/2134/2615. 


\title{
Approximations to wave propagation through doubly-periodic arrays of scatterers
}

\author{
P. McIVER \\ Department of Mathematical Sciences, Loughborough University, \\ Loughborough, Leicestershire, LE11 3TU, UK
}

\begin{abstract}
The propagation of waves through a doubly-periodic array of identical rigid scatterers is considered in the case that the field equation is the two-dimensional Helmholtz equation. The method of matched asymptotic expansions is used to obtain the dispersion relation corresponding to wave propagation through an array of scatterers of arbitrary shape that are each small relative to both the wave length and the array periodicity. The results obtained differ from those obtained from homogenization in that there is no requirement that the wave length be much smaller than the array periodicity, and hence it is possible to examine phenomena, such as band gaps, that are associated with the array periodicity.
\end{abstract}

Keywords: Propagation; Scattering.

\section{Introduction}

There is a huge literature on the propagation of waves through periodic media in many physical contexts that include acoustics, elasticity, electromagnetic theory and water waves (see [1] for a substantial on-line bibliography). An important part of this literature is concerned with cylindrical scatterers arranged in a doubly-periodic array. For example, Zalipaev et. al. [2] use a multipole expansion method to investigate wave propagation through an elastic medium containing a doubly periodic arrangement of circular holes and they obtain approximations as the wavelength becomes large relative to both the radius $a$ of the holes, and the length scale $L$ of the array periodicity. In particular, they do this in the case of anti-plane shear, for which the displacement of the elastic medium satisfies the Helmholtz equation with wave number $k$ and a homogeneous Neumann 
condition on the surfaces of the holes, and obtain simple approximate formulae for the effective refractive index and the group velocity of waves. Essentially the same approximations were obtained for scattering by rigid cylinders in the context of acoustic/water waves by Evans and Shipway [3] using a different formalism based on work by Blevins [4] in which each rigid cylinder is replaced by a suitably constrained cylinder of compressible fluid. The fundamental assumptions behind these approximations are that $k L \ll 1$ and $a / L \ll 1$ (and hence $k a \ll 1$ ) so that the problem is 'homogenized' and variations on the scales of $a$ and $L$ are removed. A particular feature of such approximations is that they are not able to describe phenomena associated with the periodicity of the array such as band gaps (wave numbers for which plane waves cannot propagate through the array). The aim here is to obtain simple approximations that do display these phenomena.

In the present work attention is restricted to the solution of the two-dimensional Helmholtz equation in the region exterior to a doubly-periodic array of identical rigid scatterers (although there is considerable scope for extending the ideas to other situations). The scatterers may be of arbitrary shape as long as the characteristic size $a$ satisfies both $k a \ll 1$ and $a / L \ll 1$, so that the scatterers are small relative to both the wave length and the array periodicity. The results given here are distinguished from those obtained through homogenization in that now $k L$ is allowed to be an order one quantity so that phenomena associated with the periodicity of the array may be described. The main idea is to obtain perturbations of plane wave solutions due to the presence of the scatterers, and the analysis is based on a scheme of matched asymptotic expansions that is closely related to previous work on the description of wave trapping within a water-filled channel [5].

\section{Formulation}

Given two-dimensional Cartesian coordinates $x$ and $y$, the aim is to find solutions for $\phi$ of the Helmholtz equation

$$
\frac{\partial^{2} \phi}{\partial x^{2}}+\frac{\partial^{2} \phi}{\partial y^{2}}+k^{2} \phi=0
$$

that satisfy the Neumann condition

$$
\frac{\partial \phi}{\partial n}=0
$$

on identical scatterers $C_{j}$ uniformly distributed in an infinite lattice $\Lambda$, where $n$ is a coordinate measured normal to $C_{j}$. Scatterer $j$ is associated with a local origin $O_{j}$ located at the lattice point

$$
\mathbf{R}_{j}=n_{1} \mathbf{a}_{1}+n_{2} \mathbf{a}_{2}, \quad n_{1}, n_{2} \in \mathbb{Z},
$$

for given independent vectors $\mathbf{a}_{1}$ and $\mathbf{a}_{2}$. In particular, solutions are sought that, for all lattice vectors, satisfy the 'Bloch condition'

$$
\phi\left(\mathbf{r}+\mathbf{R}_{j}\right)=\mathrm{e}^{\mathrm{i} \boldsymbol{\beta}^{\mathrm{T}} \mathbf{R}_{j}} \phi(\mathbf{r})
$$


where $\mathbf{r}=(x, y)^{\mathrm{T}}$ and $\boldsymbol{\beta}=\left(q_{1}, q_{2}\right)^{\mathrm{T}}$ is a prescribed vector. This is an eigenvalue problem for the Laplacian with $-k^{2}$ as the eigenvalue and the eigensolutions correspond to waves that propagate through the array.

In addition to the global coordinates, local polar coordinates $\left(r_{j}, \theta_{j}\right)$ are used with origin at $O_{j}$. The Bloch condition (4) allows the solution to be obtained through consideration of a primary lattice cell chosen to be that containing the origin $O$ of the global coordinates. The polar coordinates with origin at $O$ are denoted by $(r, \theta)$ and the scatterer associated with $O$ by $C$.

The Bloch condition (4) is satisfied by plane waves of the form

$$
\phi_{m}(\mathbf{r})=\mathrm{e}^{\mathrm{i} \boldsymbol{\beta}_{m}^{\mathrm{T}} \mathbf{r}}
$$

where

$$
\boldsymbol{\beta}_{m}=\boldsymbol{\beta}+\mathbf{K}_{m}
$$

and each

$$
\mathbf{K}_{m}=2 \pi\left(m_{1} \mathbf{b}_{1}+m_{2} \mathbf{b}_{2}\right), \quad m_{1}, m_{2} \in \mathbb{Z},
$$

is a reciprocal lattice vector with

$$
\mathbf{a}_{i}^{\mathrm{T}} \mathbf{b}_{j}=\delta_{i j}, \quad i, j=1,2
$$

The reciprocal lattice vectors have the property that, for any lattice vector $\mathbf{R}_{j}$,

$$
\mathbf{K}_{m}^{\mathrm{T}} \mathbf{R}_{j}=2 \pi p, \quad p \in \mathbb{Z}
$$

In the absence of the scatterers, each $\phi_{m}$ provides a solution to the Bloch problem provided $k$ is chosen to ensure that the field equation (1) is satisfied, in other words provided

$$
k^{2}=\beta_{m}^{2} \quad \text { where } \quad \beta_{m}=\left|\boldsymbol{\beta}_{m}\right|
$$

For example, for a square lattice of side $L$ aligned with the coordinate axes, plane-wave solutions satisfying the Bloch condition are

$$
\phi_{m}=\mathrm{e}^{\mathrm{i}\left[\left(q_{1}+2 \pi m_{1} / L\right) x+\left(q_{2}+2 \pi m_{2} / L\right) y\right]}, \quad m_{1}, m_{2} \in \mathbb{Z},
$$

and the field equation is satisfied as long as

$$
k^{2}=\left(q_{1}+2 \pi m_{1} / L\right)^{2}+\left(q_{2}+2 \pi m_{2} / L\right)^{2}
$$

The results given here for the case when scatterers are present arise from consideration of perturbations to combinations of the plane-wave solutions (5).

The method of matched asymptotic expansions is used under the assumptions that $\epsilon \equiv k a \ll 1$ and $k L$ is of order unity, where $a$ is a typical dimension of the scatterer $C$ and $L$ is a typical 
dimension of a cell within the lattice $\Lambda$. It follows that $a / L \ll 1$ so that each scatterer is much smaller than a cell of the lattice. To facilitate the solution each lattice cell is divided into two overlapping regions. For the primary cell these are an outer region at distances $r \gg a$ and an inner region within distances $r \ll k^{-1}$ of the scatterer; in the inner region a scaled coordinate $\rho=r / a$ will be used.

\section{Solution}

In the outer region, far from each scatterer, the solution is constructed from solutions of the Helmholtz equation (1) that satisfy the Bloch condition (4) and that are singular at the lattice points. Such solutions are

$$
G_{n}(k r, \theta)=\sum_{\mathbf{R}_{j} \in \Lambda} \mathrm{e}^{\mathrm{i} \boldsymbol{\beta}^{\mathrm{T}} \mathbf{R}_{j}} \mathrm{H}_{n}^{(1)}\left(k r_{j}\right) \mathrm{e}^{\mathrm{i} n \theta_{j}}, \quad n \in \mathbb{Z},
$$

and each corresponds to disturbances that propagate away from the scatterers with phases adjusted to ensure that $G_{n}$ satisfies the Bloch condition (4). By Graf's addition theorem

$$
G_{n}(k r, \theta)=\mathrm{H}_{n}^{(1)}(k r) \mathrm{e}^{\mathrm{i} n \theta}+\sum_{p}(-1)^{n-p} \sigma_{n-p} \mathrm{~J}_{p}(k r) \mathrm{e}^{\mathrm{i} p \theta}
$$

where the lattice sum

$$
\sigma_{n}=\sum_{\mathbf{R}_{j} \in \Lambda}^{\prime} \mathrm{e}^{\mathrm{i} \boldsymbol{\beta}^{\mathrm{T}} \mathbf{R}_{j}} \mathrm{H}_{n}^{(1)}\left(k R_{j}\right) \mathrm{e}^{\mathrm{i} n \alpha_{j}}
$$

and the dash indicates that $\mathbf{R}_{j}=\mathbf{0}$ is omitted from the summation.

Angles $\tau_{m}$ are defined through

$$
\boldsymbol{\beta}_{m}=\beta_{m} \mathbf{e}_{1 m}
$$

where

$$
\mathbf{e}_{p m}=\left(\begin{array}{c}
\cos p \tau_{m} \\
\sin p \tau_{m}
\end{array}\right) .
$$

The lattice sums have simple poles at $k= \pm \beta_{m}$ (see [6], for example) and, in particular, for each unique vector $\boldsymbol{\beta}_{m}$

$$
\sigma_{n} \sim \frac{4 \mathrm{i}^{n+1} \mathrm{e}^{\mathrm{i} n \tau_{m}}}{A\left(k^{2}-\beta_{m}^{2}\right)} \quad \text { as } \quad k^{2} \rightarrow \beta_{m}^{2}
$$

where $A$ is the area of one cell of the lattice. The poles of the lattice sums correspond to the plane wave solutions (5) that exist in the absence of the scatterers. There may be $M \geq 1$ distinct vectors $\boldsymbol{\beta}_{m}$ with the same magnitude $\beta_{m}$ and, with this in mind, the lattice sums are written

$$
\sigma_{n}=\sum_{m=1}^{M} \frac{\sigma_{n m}^{(1)}}{\left(k^{2}-\beta_{m}^{2}\right) L^{2}}+\sigma_{n}^{(2)}
$$


where

$$
\sigma_{n m}^{(1)}=\frac{4 \mathrm{i}^{n+1} \mathrm{e}^{\mathrm{i} n \tau_{m}}}{A / L^{2}}
$$

and $\sigma_{n}^{(2)}$ is an analytic function of $k$ within neighbourhoods of $k= \pm \beta_{m}$.

Solutions are sought for $k$ in a neighbourhood of $\beta_{m}$ and it is assumed here that

$$
\left(k^{2}-\beta_{m}^{2}\right) L^{2}=\epsilon^{2} \delta_{m}
$$

where $\delta_{m}$ is strictly of order one in $\epsilon$ for $k^{2} \neq \beta_{m}^{2}$. It is not necessary to make the specific assumption (21), the order in $\epsilon$ of $k^{2}-\beta_{m}^{2}$ can be obtained as part of the solution. However, equation (21) is adopted now in order to simplify the presentation and it is consistent with previous work concerned with wave motion around small objects [5] (the matching would fail if the relation were incorrect). In view of (21), the matching may be carried out more conveniently if the singular solutions of the Helmholtz equation defined in (13) are modified to

$$
g_{n}(k r, \theta)=\epsilon^{2} G_{n}(k r, \theta)=g_{n}^{(1)}(k r, \theta)+\epsilon^{2} g_{n}^{(2)}(k r, \theta)
$$

where

$$
g_{n}^{(1)}(k r, \theta)=\sum_{m=1}^{M} \frac{1}{\delta_{m}} \sum_{p}(-1)^{n-p} \sigma_{n-p, m}^{(1)} \mathrm{J}_{p}(k r) \mathrm{e}^{\mathrm{i} p \theta}=(-1)^{n} \sum_{m=1}^{M} \frac{\sigma_{n m}^{(1)}}{\delta_{m}} \mathrm{e}^{\mathrm{i} k r \cos \left(\theta-\tau_{m}\right)}
$$

and

$$
g_{n}^{(2)}(k r, \theta)=\mathrm{H}_{n}^{(1)}(k r) \mathrm{e}^{\mathrm{i} n \theta}+\sum_{p}(-1)^{n-p} \sigma_{n-p}^{(2)} \mathrm{J}_{p}(k r) \mathrm{e}^{\mathrm{i} p \theta}
$$

(this modification ensures that $\epsilon$ does not appear in the denominator of any expression).

As the boundary-value problem is homogeneous, the leading-order outer solution may be taken as strictly order one in $\epsilon$ and written

$$
\Psi^{(0)}=\sum_{n} A_{n} g_{n}^{(1)}(k r, \theta)
$$

where $\Psi^{(m)}$ denotes the outer solution up to terms in $\epsilon^{m}$. Note that each $g_{n}^{(1)}(k r, \theta)$ is a sum over plane waves of the type given in (5), and hence the leading-order outer solution is a combination of such plane waves. From (23)

$$
\Psi^{(0,1)}=\sum_{n} A_{n}(-1)^{n} \sum_{m=1}^{M} \frac{\sigma_{n m}^{(1)}}{\delta_{m}}\left[1+\mathrm{i} \epsilon \rho \mathbf{e}_{1 m}^{\mathrm{T}}\left(\begin{array}{c}
\cos \theta \\
\sin \theta
\end{array}\right)\right]
$$

where $\Psi^{(m, l)}$ denotes the expansion up to $\epsilon^{l}$ of $\Psi^{(m)}$ after it is written in terms of the inner coordinate $\rho$. In the inner region, the inner solution up to terms in $\epsilon^{l}$ is denoted by $\psi^{(l)}$ and $\psi^{(l, m)}$ denotes its expansion up to $\epsilon^{m}$ after it is written in terms of the outer coordinate $k r$. Matching is enforced by requiring $\Psi^{(m, l)} \equiv \psi^{(l, m)}$ for every $m$ and $l[7]$. 
In terms of the inner variables the field equation for the inner solution $\psi$ is

$$
\frac{1}{\rho} \frac{\partial}{\partial \rho}\left(\rho \frac{\partial \psi}{\partial \rho}\right)+\frac{1}{\rho^{2}} \frac{\partial^{2} \psi}{\partial \theta^{2}}+\epsilon^{2} \psi=0
$$

The form of $\Psi^{(0,1)}$ indicates an inner development

$$
\psi^{(1)}=\psi_{0}+\epsilon \psi_{1}
$$

where, from equating powers of $\epsilon$ in the field equation (27) and boundary condition (2), both $\psi_{0}$ and $\psi_{1}$ are harmonic functions that satisfy the homogeneous Neumann condition on $C$. The inner expansion $\Psi^{(0,1)}$ 'drives' a flow past the scatterer $C$ and to effect the matching it is necessary to take

$$
\psi^{(1)}=B_{0}+\epsilon\left\{B_{1}+\mathbf{u}_{1}^{\mathrm{T}}\left[\rho\left(\begin{array}{c}
\cos \theta \\
\sin \theta
\end{array}\right)+\chi_{1}(\rho, \theta)\right]\right\}
$$

here, from (2),

$$
\begin{gathered}
\frac{\partial \boldsymbol{\chi}_{1}}{\partial n}=-\frac{\partial}{\partial n}\left[\rho\left(\begin{array}{c}
\cos \theta \\
\sin \theta
\end{array}\right)\right] \text { on } C, \\
\boldsymbol{\chi}_{1}-\frac{\boldsymbol{M}}{\rho}\left(\begin{array}{c}
\cos \theta \\
\sin \theta
\end{array}\right)=o\left(\rho^{-1}\right) \text { as } \quad \rho \rightarrow \infty
\end{gathered}
$$

and

$$
\boldsymbol{M}=\left(\begin{array}{ll}
\mu_{11} & \mu_{12} \\
\mu_{21} & \mu_{22}
\end{array}\right)
$$

is the matrix of dipole coefficients determined by the shape of the scatterer [8, p. 127]. As the solution to a homogeneous problem is sought the choice $B_{0}=1$ is made without loss of generality. The outer expansion of the leading order inner solution $\psi^{(1)}$ has terms no more singular than a dipole, and hence cannot be matched to higher singularities in the outer solution; thus

$$
A_{n}=0, \quad|n| \geq 2
$$

and now

$$
\Psi^{(0,1)}=\sum_{n=-1}^{1} A_{n}(-1)^{n} \sum_{m=1}^{M} \frac{\sigma_{n m}^{(1)}}{\delta_{m}}\left[1+\mathrm{i} \epsilon \rho \mathbf{e}_{1 m}^{\mathrm{T}}\left(\begin{array}{c}
\cos \theta \\
\sin \theta
\end{array}\right)\right] .
$$

From (29)

$$
\psi^{(1,0)}=1+\epsilon\left\{B_{1}+\rho \mathbf{u}_{1}^{\mathrm{T}}\left(\begin{array}{c}
\cos \theta \\
\sin \theta
\end{array}\right)\right\}
$$

and the matching of $\Psi^{(0,1)}$ with $\psi^{(1,0)}$ yields

$$
\sum_{n=-1}^{1} A_{n}(-1)^{n} \sum_{m=1}^{M} \frac{\sigma_{n m}^{(1)}}{\delta_{m}}=1
$$


and

$$
\mathbf{u}_{1}=\mathrm{i} \sum_{n=-1}^{1} A_{n}(-1)^{n} \sum_{m=1}^{M} \frac{\sigma_{n m}^{(1)}}{\delta_{m}} \mathbf{e}_{1 m} .
$$

The outer solution is continued as

$$
\Psi^{(2)}=\sum_{n=-1}^{1} A_{n}\left\{g_{n}^{(1)}(k r, \theta)+\epsilon^{2} g_{n}^{(2)}(k r, \theta)\right\}+\epsilon \sum_{n} C_{n} g_{n}^{(1)}(k r, \theta)+\epsilon^{2} \sum_{n} D_{n} g_{n}^{(1)}(k r, \theta)
$$

(possible intermediate terms involving $\log \epsilon$ prove impossible to match and are omitted) which has an inner expansion

$$
\begin{aligned}
\Psi^{(2,2)} & =\sum_{n=-1}^{1} A_{n}(-1)^{n} \sum_{m=1}^{M} \frac{\sigma_{n m}^{(1)}}{\delta_{m}}\left\{1+\mathrm{i} \epsilon \rho \mathbf{e}_{1 m}^{\mathrm{T}}\left(\begin{array}{c}
\cos \theta \\
\sin \theta
\end{array}\right)-\frac{1}{4} \epsilon^{2} \rho^{2}\left[1+\mathbf{e}_{2 m}^{\mathrm{T}}\left(\begin{array}{c}
\cos 2 \theta \\
\sin 2 \theta
\end{array}\right)\right]\right\} \\
& +\epsilon^{2}\left\{A_{0}\left[1+\frac{2 \mathrm{i}}{\pi}\left(\log \frac{\epsilon \rho}{2}+\gamma\right)+\sigma_{0}^{(2)}\right]+A_{1}\left[-\frac{2 \mathrm{i}}{\pi \epsilon \rho} \mathrm{e}^{\mathrm{i} \theta}-\sigma_{1}^{(2)}\right]+A_{-1}\left[\frac{2 \mathrm{i}}{\pi \epsilon \rho} \mathrm{e}^{-\mathrm{i} \theta}-\sigma_{-1}^{(2)}\right]\right\} \\
& +\sum_{n} C_{n}(-1)^{n} \sum_{m=1}^{M} \frac{\sigma_{n m}^{(1)}}{\delta_{m}}\left[1+\mathrm{i} \epsilon \rho \mathbf{e}_{1 m}^{\mathrm{T}}\left(\begin{array}{c}
\cos \theta \\
\sin \theta
\end{array}\right)\right]+\sum_{n} D_{n}(-1)^{n} \sum_{m=1}^{M} \frac{\sigma_{n m}^{(1)}}{\delta_{m}} .
\end{aligned}
$$

This inner expansion indicates that the inner solution must be continued as

$$
\psi^{(2)}=1+\epsilon\left\{B_{1}+\mathbf{u}_{1}^{\mathrm{T}}\left[\rho\left(\begin{array}{c}
\cos \theta \\
\sin \theta
\end{array}\right)+\boldsymbol{\chi}_{1}(\rho, \theta)\right]\right\}+\epsilon^{2} \log \epsilon E_{0}+\epsilon^{2} \psi_{2}
$$

where, from (27), $\psi_{2}$ satisfies the field equation

$$
\frac{1}{\rho} \frac{\partial}{\partial \rho}\left(\rho \frac{\partial \psi_{2}}{\partial \rho}\right)+\frac{1}{\rho^{2}} \frac{\partial^{2} \psi_{2}}{\partial \theta^{2}}=-1
$$

and also the homogeneous boundary condition

$$
\frac{\partial \psi_{2}}{\partial n}=0 \quad \text { on } \quad C
$$

The appropriate form required to effect the matching with $\Psi^{(2,2)}$ is

$$
\psi_{2}=-\frac{1}{4} \rho^{2}+\Omega(\rho, \theta)+F_{0}+\mathbf{u}_{2}^{\mathrm{T}}\left[\rho\left(\begin{array}{c}
\cos \theta \\
\sin \theta
\end{array}\right)+\chi_{1}(\rho, \theta)\right]+\mathbf{v}_{2}^{\mathrm{T}}\left[\rho^{2}\left(\begin{array}{c}
\cos 2 \theta \\
\sin 2 \theta
\end{array}\right)+\chi_{2}(\rho, \theta)\right],
$$

where $\Omega$ is a harmonic function introduced to compensate for the flux across $C$ that is induced by the first term in (43), and $\chi_{2}(\rho, \theta)=o(1)$ as $\rho \rightarrow \infty$. Calculation of the flux across $C$ shows that as $\rho \rightarrow \infty$ it is required that

$$
\Omega(\rho, \theta)-\frac{S}{2 \pi a^{2}} \log \rho \rightarrow 0
$$


where $S$ is the area contained within $C$. It follows immediately that

$$
\begin{aligned}
\psi^{(2,2)} & =1+\epsilon\left\{B_{1}+\mathbf{u}_{1}^{\mathrm{T}}\left[\rho\left(\begin{array}{c}
\cos \theta \\
\sin \theta
\end{array}\right)+\frac{\boldsymbol{M}}{\rho}\left(\begin{array}{c}
\cos \theta \\
\sin \theta
\end{array}\right)\right]\right\}+\epsilon^{2} \log \epsilon E_{0} \\
& +\epsilon^{2}\left\{-\frac{1}{4} \rho^{2}+\frac{S}{2 \pi a^{2}} \log \rho+F_{0}+\rho \mathbf{u}_{2}^{\mathrm{T}}\left(\begin{array}{c}
\cos \theta \\
\sin \theta
\end{array}\right)+\rho^{2} \mathbf{v}_{2}^{\mathrm{T}}\left(\begin{array}{c}
\cos 2 \theta \\
\sin 2 \theta
\end{array}\right)\right\}
\end{aligned}
$$

and matching with $\Psi^{(2,2)}$ gives, in particular,

$$
A_{0}=-\frac{\mathrm{i} S}{4 a^{2}} \quad \text { and } \quad\left(A, A_{-1}\right)=\frac{\pi}{4 \mathrm{i}} \mathbf{u}_{1}^{\mathrm{T}} \boldsymbol{M}\left(\begin{array}{cc}
-1 & 1 \\
\mathrm{i} & \mathrm{i}
\end{array}\right) .
$$

With the values of $A_{n}$ given by (46), equation (36) is

$$
\frac{\pi L^{2}}{A} \sum_{m=1}^{M} \frac{1}{\delta_{m}}\left[\frac{S}{\pi a^{2}}+2 \mathrm{i} \mathbf{u}_{1}^{\mathrm{T}} \boldsymbol{M} \mathbf{e}_{1 m}\right]=1
$$

and equation (37) is

$$
\mathbf{u}_{1}=\frac{\pi \mathrm{i} L^{2}}{A} \sum_{m=1}^{M} \frac{\mathbf{e}_{1 m}}{\delta_{m}}\left[\frac{S}{\pi a^{2}}+2 \mathrm{i} \mathbf{u}_{1}^{\mathrm{T}} \boldsymbol{M} \mathbf{e}_{1 m}\right] .
$$

Equation (48) may be solved for $\mathbf{u}_{1}$ and then equation (47) yields, for a given $\boldsymbol{\beta}$, the relation between the wave number $k$ and the geometry of the scatterer $C$. An alternative is to introduce

$$
U_{m}=\frac{1}{\delta_{m}}\left[1+\frac{2 \mathrm{i} \pi a^{2}}{S} \mathbf{u}_{1}^{\mathrm{T}} \boldsymbol{M} \mathbf{e}_{1 m}\right]
$$

and rewrite equation (48) as

$$
\delta_{p} U_{p}+\frac{2 \pi L^{2}}{A} \sum_{m=1}^{M}\left[\mathbf{e}_{1 m}^{\mathrm{T}} \boldsymbol{M} \mathbf{e}_{1 m}\right] U_{m}=1, \quad p=1,2, \ldots M .
$$

Equation (50) is readily solved for $\left\{U_{m}: m=1,2, \ldots M\right\}$ and then equation (47) in the form

$$
\frac{S L^{2}}{A a^{2}} \sum_{m=1}^{M} U_{m}=1
$$

again yields the wave number $k$. Calculations (both symbolic and numerical) for this paper were carried out using the computer algebra package Mathematica and it is this second method that proved to be the more efficient. With each method the same polynomial of degree $M$ in $k^{2}$ is obtained. The geometry of the lattice appears through the reciprocal lattice vectors in the definitions of each $\delta_{m}$ and $\mathbf{e}_{1 m}$. 


\section{Results}

In the case $M=1$ equations (50) and (51) reduce to

$$
\delta_{1}=\frac{\pi L^{2}}{A}\left(\frac{S}{\pi a^{2}}-2 \mathbf{e}_{11}^{\mathrm{T}} \boldsymbol{M} \mathbf{e}_{11}\right)
$$

or

$$
k^{2}=\beta_{1}^{2}\left\{1-\frac{\pi a^{2}}{A}\left(\frac{S}{\pi a^{2}}-2 \mathbf{e}_{11}^{\mathrm{T}} \boldsymbol{M} \mathbf{e}_{11}\right)\right\}^{-1}
$$

and, for a given $\boldsymbol{\beta}$, this shows explicitly how $k$ depends upon the geometry of the scatterer through $S$ and $\boldsymbol{M}$. This generalizes the relations obtained by other authors using homogenization to any region of the parameter space that is in the neighbourhood of a single pole at $k=\beta_{1}$. For a scatterer that is symmetric about the two coordinate axes $\mu_{12}=\mu_{21}=0$ and (53) reduces to

$$
k^{2}=\beta_{1}^{2}\left\{1-\frac{\pi a^{2}}{A}\left(\frac{S}{\pi a^{2}}-2\left[\mu_{11} \cos ^{2} \tau_{1}+\mu_{22} \sin ^{2} \tau_{1}\right]\right)\right\}^{-1} .
$$

In some homogenization schemes the results have been expressed in terms of added mass coefficients $\mathrm{a}_{i i}$ rather than dipole coefficients $\mu_{i i}$; the two are related by

$$
\mathrm{a}_{i i}=\rho\left(2 \pi \mu_{i i} a^{2}-S\right)
$$

$[9$, p. 144]. In terms of the added mass $(54)$ is

$$
k^{2}=\beta_{1}^{2}\left\{1+\frac{S}{A}\left[\frac{\mathrm{a}_{11}}{\rho S} \cos ^{2} \tau_{1}+\frac{\mathrm{a}_{22}}{\rho S} \sin ^{2} \tau_{1}\right]\right\}^{-1} .
$$

In the case that $C$ is a circle of radius $a, \mathrm{a}_{11}=\mathrm{a}_{22}=\rho S$ and hence

$$
k^{2}=\beta_{1}^{2}\left[1+\frac{\pi a^{2}}{A}\right]^{-1} \text {. }
$$

These results may be compared with results obtained elsewhere for the lowest mode, that is when

$$
\boldsymbol{\beta}_{1}=\boldsymbol{\beta} \equiv \beta\left(\begin{array}{c}
\cos \tau \\
\sin \tau
\end{array}\right) .
$$

In this case, Evans and Shipway [3] give the model field equation

$$
\left[1+\frac{S}{A} \frac{\mathrm{a}_{11}}{\rho S}\right]^{-1} \frac{\partial^{2} \phi}{\partial x^{2}}+\left[1+\frac{S}{A} \frac{\mathrm{a}_{22}}{\rho S}\right]^{-1} \frac{\partial^{2} \phi}{\partial y^{2}}+k^{2} \phi=0
$$

and seeking solutions in the form

$$
\phi=\mathrm{e}^{\mathrm{i} \beta(x \cos \tau+y \sin \tau)}
$$

yields

$$
k^{2}=\beta^{2}\left\{\cos ^{2} \tau\left[1+\frac{S}{A} \frac{\mathrm{a}_{11}}{\rho S}\right]^{-1}+\sin ^{2} \tau\left[1+\frac{S}{A} \frac{\mathrm{a}_{22}}{\rho S}\right]^{-1}\right\} .
$$




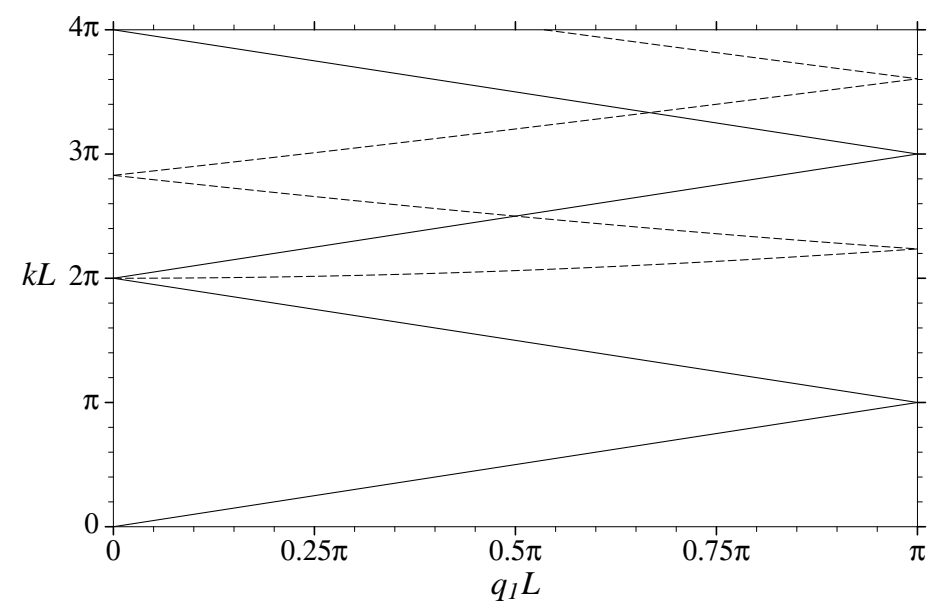

Figure 1: Positions of poles of the lattice sums in wave number space when $q_{2}=0$; single pole $(-)$, two poles $(----)$.

However, by assumption $S / A \ll 1$ and expansion of both of equations (56) and (61) in powers of $S / A$ yields the same approximation, namely

$$
k^{2}=\beta^{2}\left\{1-\frac{S}{A}\left[\frac{\mathrm{a}_{11}}{\rho S} \cos ^{2} \tau+\frac{\mathrm{a}_{22}}{\rho S} \sin ^{2} \tau\right]\right\} .
$$

The theory for $M>1$ will be illustrated in detail through a comparison of scatterers that are either circles or ellipses arranged in a square lattice with cells of side $L$. The circles have radius $a$ and the ellipse semi-axes of length $a$ and $b$ parallel to the $x$ and $y$ axes respectively. For the ellipse the matrix of dipole coefficients is

$$
\boldsymbol{M}=\left(\begin{array}{cc}
\frac{b(a+b)}{2 a^{2}} & 0 \\
0 & \frac{a+b}{2 a}
\end{array}\right)
$$

[9, p. 145] and $S=\pi a b$. For the case $q_{2}=0$ the positions of the poles of the lattice sums are shown in figure 1 using a reduced zone scheme in which $q_{1} \in[0, \pi]$ and all poles are obtained from $\pm q_{1}+2 n \pi / L, n \in \mathbb{Z}$. The solid lines show the points in wave number space at which the lattice sums have a single pole and the dashed lines correspond to points for which there are two poles. At the intersections of these lines there may be up to four poles of the lattice sums. As remarked earlier, in the absence of the scatterers the poles correspond to the plane waves (11) that satisfy the Bloch condition (4).

Attention will next be focused upon $\left(q_{1} L, q_{2} L, k L\right)=(\pi, 0, \pi)$ for which there are two poles and for calculations within a neighbourhood of this point the forms

$$
\boldsymbol{\beta}_{1} L=\left(q_{1} L, q_{2} L\right)^{\mathrm{T}} \quad \text { and } \quad \boldsymbol{\beta}_{2} L=\left(q_{1} L-2 \pi, q_{2} L\right)^{\mathrm{T}}
$$



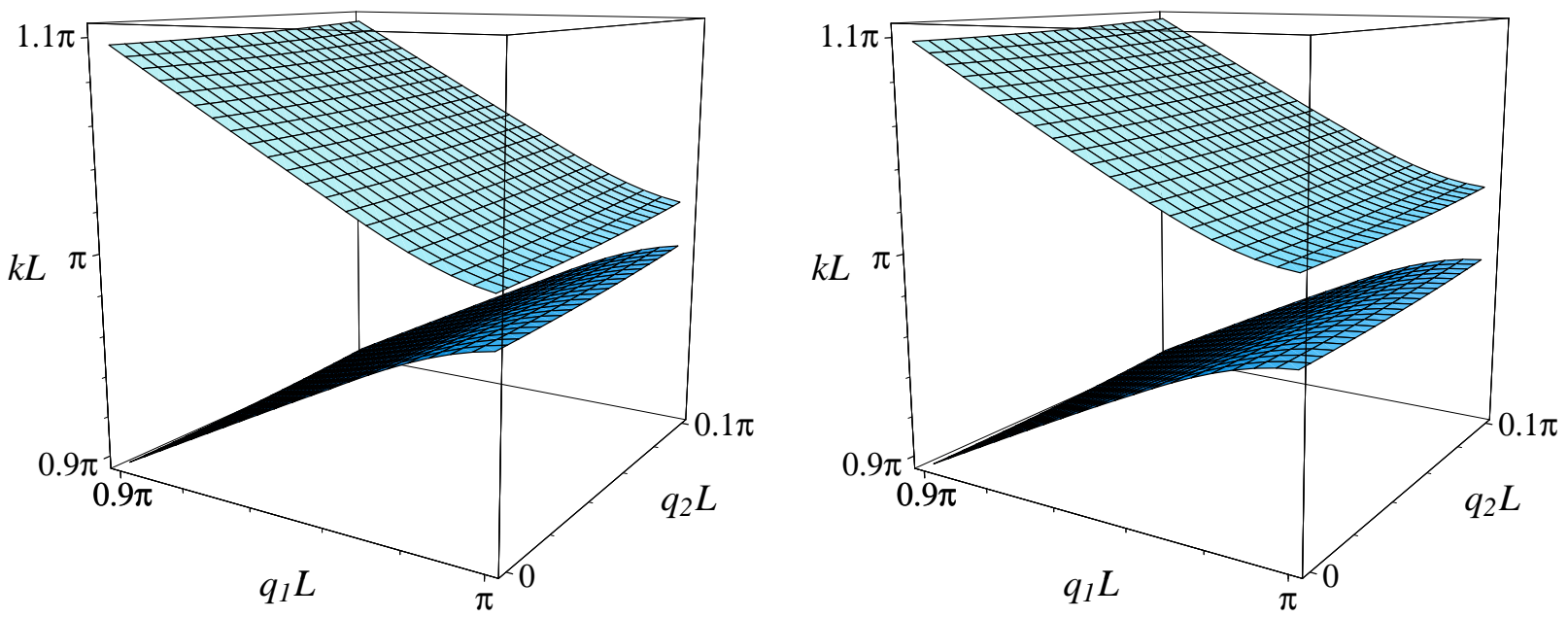

Figure 2: Solution surfaces in the vicinity of $\left(q_{1} L, q_{2} L, k L\right)=(\pi, 0, \pi)$; circle of radius $a=L / 20$ (left), ellipse with semi-axes $a=L / 10, b=L / 20$ (right).

are appropriate (because, when $\left(q_{1} L, q_{2} L\right)=(\pi, 0), \beta_{1} L=\beta_{2} L=\pi \equiv k L$ and the lattice sums are singular). With these $\beta_{m}$, and for $q_{2}=0$, equations (50) and (51) reduce to

$$
\begin{array}{r}
{\left[1+\frac{2 \pi b^{2}}{L^{2}}\left(1-\frac{2 \pi a}{L}\left(\frac{a}{L}+\frac{b}{L}\right)\right)\right](k L)^{4}-2\left(1+\frac{\pi b^{2}}{L^{2}}\right)\left[\left(q_{1} L\right)^{2}-2 \pi q_{1} L+2 \pi^{2}\right](k L)^{2}} \\
+\left(q_{1} L\right)^{2}\left(q_{1} L-2 \pi\right)^{2}=0 .
\end{array}
$$

When $q_{1} L=\pi$ the positive roots of the last equation are

$$
k L=\frac{\pi}{\sqrt{1+2 \pi b(a+b) / L^{2}}}, \frac{\pi}{\sqrt{1-2 \pi a b / L^{2}}}
$$

which illustrates explicitly the appearance of a band gap (at least for $q_{2}=0$ ) as the lengths of the axes of the ellipse are increased from zero. The solution in the vicinity of $\left(q_{1} L, q_{2} L, k L\right)=(\pi, 0, \pi)$ is illustrated in figure 2 where elliptical and circular scatterers are compared. The band gap is wider for the ellipse (which has a larger area), but the overall structure of the solution is very similar.

At $\left(q_{1} L, q_{2} L, k L\right)=(\pi / 2,0,5 \pi / 2)$ there are three poles of the lattice sums and within some neighbourhood of this point the appropriate forms for the $\boldsymbol{\beta}_{m}$ are

$$
\boldsymbol{\beta}_{1} L=\left(q_{1} L+2 \pi, q_{2} L\right)^{\mathrm{T}}, \quad \boldsymbol{\beta}_{2} L=\left(q_{1} L-2 \pi, q_{2} L+2 \pi\right)^{\mathrm{T}} \quad \text { and } \quad \boldsymbol{\beta}_{3} L=\left(q_{1} L-2 \pi, q_{2} L-2 \pi\right)^{\mathrm{T}}
$$

the solution in the vicinity of $\left(q_{1} L, q_{2} L, k L\right)=(\pi / 2,0,5 \pi / 2)$ is shown in figure 3 . For $\left(q_{1} L, q_{2} L, k L\right)=$ $(0,0,2 \pi)$ there are four poles of the lattice sums and within some neighbourhood

$$
\boldsymbol{\beta}_{1,2} L=\left(q_{1} L \pm 2 \pi, q_{2} L\right)^{\mathrm{T}} \quad \text { and } \quad \boldsymbol{\beta}_{3,4} L=\left(q_{1} L, q_{2} L \pm 2 \pi\right)^{\mathrm{T}}
$$



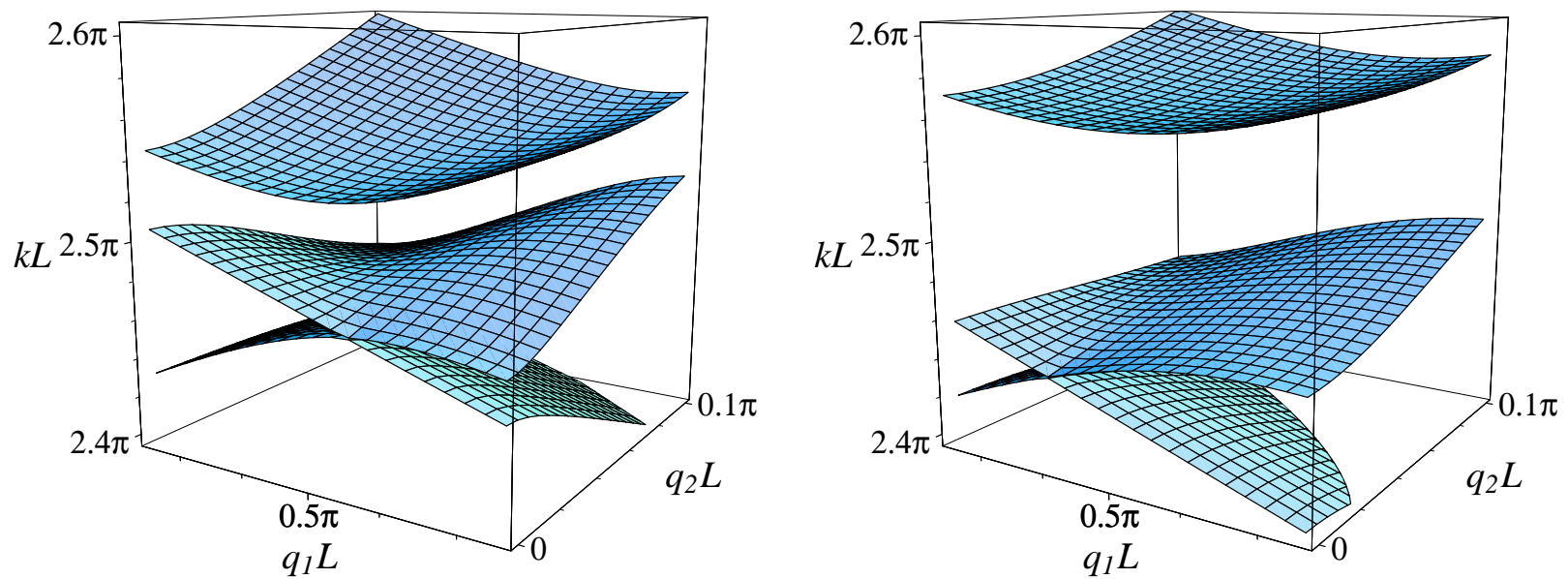

Figure 3: Solution surfaces in the vicinity of $\left(q_{1} L, q_{2} L, k L\right)=(\pi / 2,0,5 \pi / 2)$; circle of radius $a=$ $L / 20$ (left), ellipse with semi-axes $a=L / 10, b=L / 20$ (right).
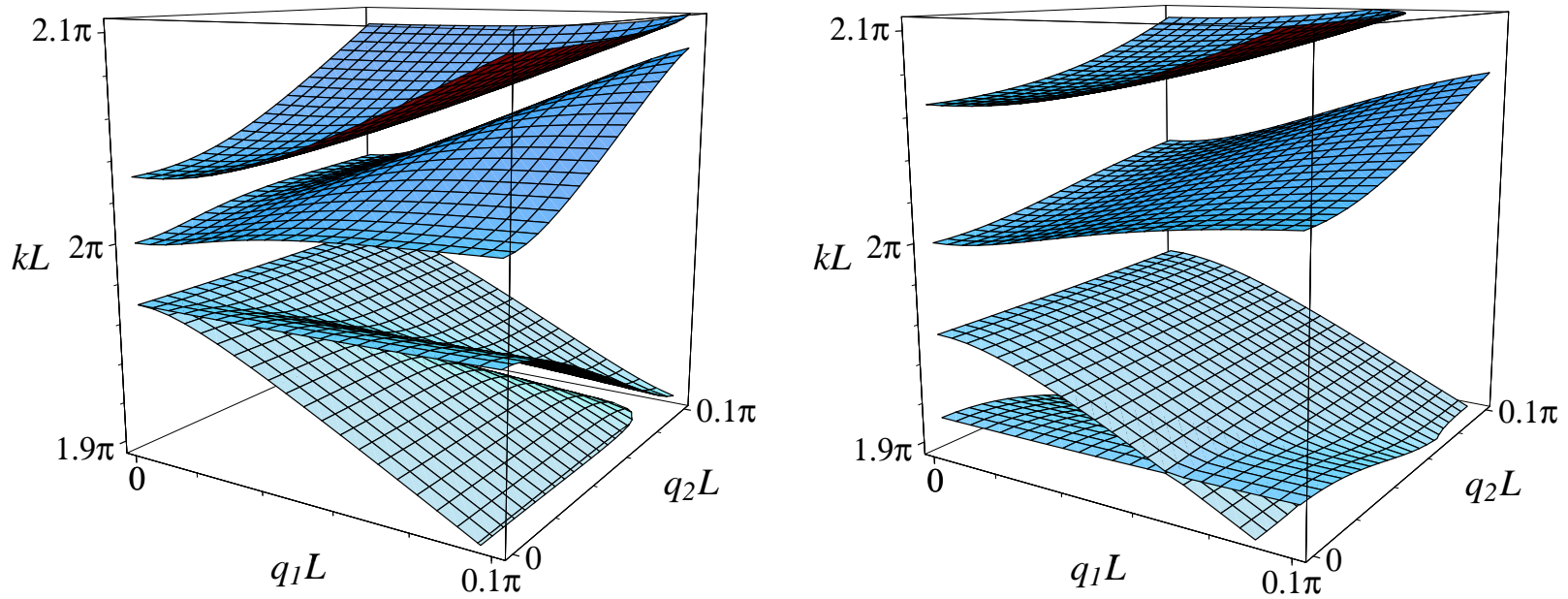

Figure 4: Solution surfaces in the vicinity of $\left(q_{1} L, q_{2} L, k L\right)=(0,0,2 \pi)$; circle of radius $a=L / 20$ (left), ellipse with semi-axes $a=L / 10, b=L / 20$ (right).

the solution in the vicinity of $\left(q_{1} L, q_{2} L, k L\right)=(0,0,2 \pi)$ is shown in figure 4 . In both these last two cases the structure of the solution for the ellipse is distinguished from that for the circle in that the diabolical points (where the sheets in wave number space touch [10]) are moved away from the points of symmetry at $q_{1} L=0, \pi / 2$.

The accuracy of the approximations given above is indicated in figure 5 through three comparisons with numerical calculations for a circle made with the Rayleigh-Ritz method [11]. In each 

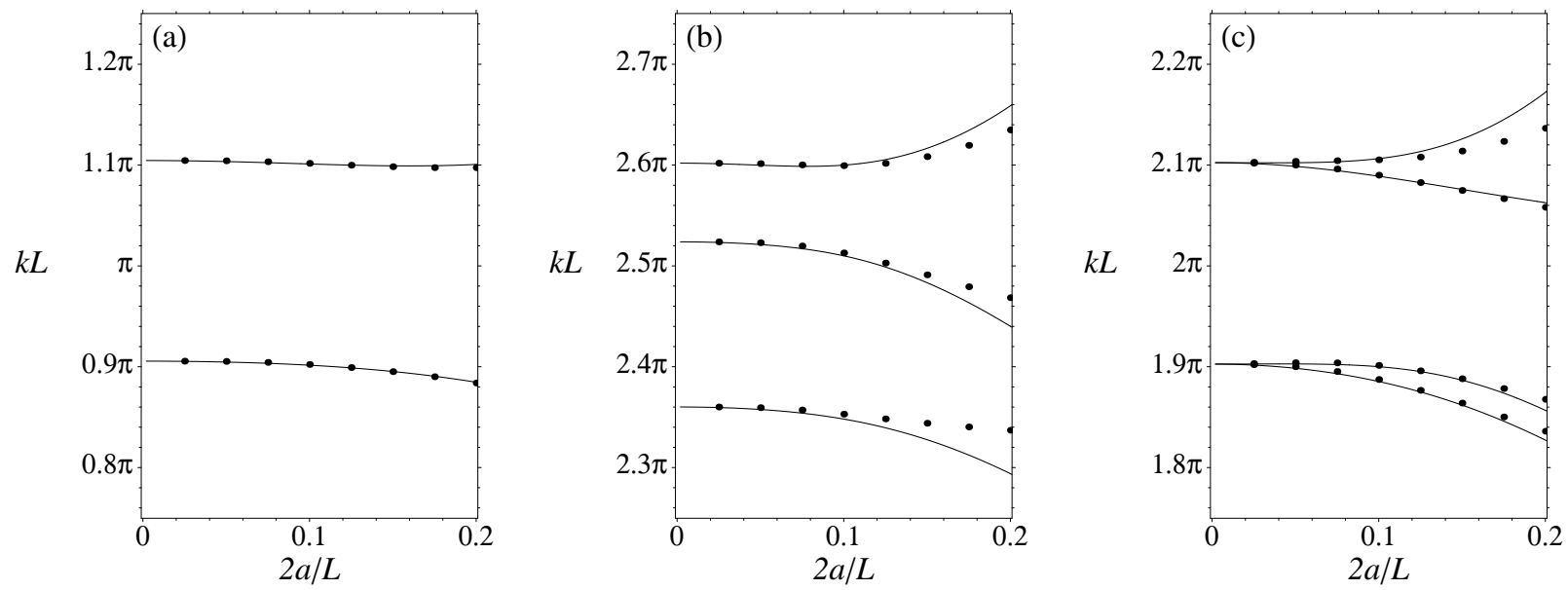

Figure 5: Comparison of the present approximations (__ $)$ with numerical calculations $(\bullet \bullet \bullet \bullet)$ for a circle of diameter $2 a$. (a) $\left(q_{1} L, q_{2} L\right)=\left(\frac{9}{10} \pi, \frac{1}{10} \pi\right)$, (b) $\left(q_{1} L, q_{2} L\right)=\left(\frac{3}{5} \pi, \frac{1}{10} \pi\right)$, (c) $\left(q_{1} L, q_{2} L\right)=$ $\left(\frac{1}{10} \pi, \frac{1}{10} \pi\right)$.

case the wave number $k L$ is given as a function of $2 a / L$ for values of $\boldsymbol{\beta} L=\left(q_{1} L, q_{2} L\right)$ whose values differ by $(\pi / 10, \pi / 10)$ from those corresponding to the poles considered above. As $k L$ increases for a fixed $2 a / L$ then the parameter $k a$, which is assumed to be small, also increases. The results in figure 1 suggest that reasonable accuracy is obtained for values of $k a$ up to about 0.4 (roughly corresponding to, for example, $2 a / L=0.1$ and $k L=2.6 \pi)$. The results shown in figures $2-4$ go beyond this value of $k a$ to more clearly show the phenomena under discussion.

\section{Conclusion}

A technique has been described that yields approximations to the dispersion relation for wave propagation through a doubly-periodic array of small scatterers of arbitrary shape. More specifically, the results show how the wave number of a plane wave is perturbed by the introduction of the scatterers. An important feature of these approximations is that they display phenomena, such as the appearance of band gaps, that are associated with the periodicity of the array. Although the method has been applied here only to rigid scatterers within a medium governed by the twodimensional Helmholtz equation, many extensions are possible. For example, the method may be applied in three dimensions and/or to scatterers that are not rigid. 


\section{Acknowledgement}

The author is grateful to Professor C.M. Linton for making available his notes on multipole expansions in Bloch problems.

\section{References}

[1] Dowling, J.P., 2006, Photonic and sonic band-gap bibliography. Available on line at: http://phys.lsu.edu/ jdowling/pbgbib.html.

[2] Zalipaev, V. V., Movchan, A.B., Poulton, C.G. and McPhedran, R.C., 2002, Elastic waves and homogenization in oblique periodic structures. Proceedings of the Royal Society of London A, 458, 1887-1912.

[3] Evans, D. V. and Shipway, B. B., 2000, A continuum model for multi-column structures in waves. Proceedings of the 15th International Workshop on Water Waves and Floating Bodies, held in Caesarea, Israel, 27 February - 1 March 2000, 47-50.

[4] Blevins, R.D., 1986, Acoustic modes of heat exchanger tube bundles. Journal of Sound and Vibration, 109, 19-31.

[5] McIver, P., 1991, Trapping of surface water waves by fixed bodies in a channel. Quarterly Journal of Mechanics and Applied Mathematics, 44, 193-208.

[6] Chin, S.K., Nicorovici, N.A. and McPhedran, R.C., 1994, Green's function and lattice sums for electromagnetic scattering by a square array of cylinders. Physical Review E, 49, 4590-4602.

[7] Crighton, D.G. and Leppington, F.G., 1973, Singular perturbation methods in acoustics: diffraction by a plate of finite thickness. Proceedings of the Royal Society of London A, 335, 313-339.

[8] Batchelor, G.K., 1967, An Introduction to Fluid Dynamics (Cambridge University Press).

[9] Newman, J. N., 1977, Marine Hydrodynamics (Cambridge, Massachusetts: MIT Press).

[10] Berry, M.V. and Wilkinson, M., 1984, Diabolical points in the spectra of triangles. Proceedings of the Royal Society of London A, 392, 15-43.

[11] McIver, P., 2000, Water-wave propagation through an infinite array of cylindrical structures. Journal of Fluid Mechanics, 424, 101-125. 